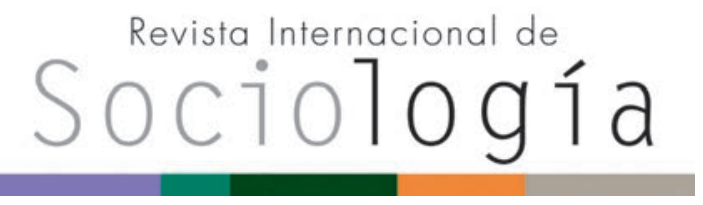

Revista Internacional de Sociología RIS

vol. 76 (1), e087, enero-marzo, 2018, ISSN-L:0034-9712 https://doi.org/10.3989/ris.2018.76.1.16.23

\section{PROSTITUCIÓN DE MENORES EN LOCALES REGISTRADOS EN MÉXICO}

\author{
SimÓN PEDRo IzCARA PALACIOS \\ Universidad Autónoma de Tamaulipas, México \\ sizcara@uat.edu.mx \\ ORCID iD: https://orcid.org/0000-0003-0523-305X
}

Cómo citar este artículo / Citation: Izcara Palacios, S. P. 2018. "Prostitución de menores en locales registrados en México". Revista Internacional de Sociología 76(1): e087. https://doi.org/10.3989/ris.2018.76.1.16.23

\section{Resumen}

En México muchas menores trabajan en establecimientos registrados donde se realiza el comercio sexual, como cantinas, bares o centros nocturnos. Este artículo, basado en una metodología cualitativa que incluyó la realización de entrevistas en profundidad en nueve estados de México a dueños de 73 locales dedicados al comercio sexual, examina la prostitución de menores en este tipo de establecimientos a través de las apreciaciones de los proxenetas, y concluye que la generalización de una cultura del soborno en la industria de la prostitución conduce a la solidificación de redes de complicidad entre las autoridades policiales y los proxenetas que favorecen la prostitución de menores.

\section{Palabras Clave}

Centros de comercio sexual; Corrupción; Trata de personas.

\section{PROSTITUTION OF MINORS IN REGISTERED PLACES IN MEXICO}

Copyright: (C) 2018 CSIC. Este es un artículo de acceso abierto distribuido bajo los términos de la licencia de uso y distribución Creative Commons Reconocimiento 4.0 Internacional (CC BY 4.0).

Recibido: 10/02/2016. Aceptado: 01/07/2016. Publicado online: 22/03/2018

\section{Abstract}

Many children in Mexico are working in registered plac-es where the sex trade is allowed, such as canteens, bars or nightclubs. This article, based on a qualitative methodology that includes in-depth interviews with owners of 73 centers dedicated to the sexual commerce in nine Mexican States, examines the prostitution of mi-nors in these places towards the appreciations of the pimps, and concludes that the spread of a culture of bribery in the prostitution industry leads to the solidifica-tion of networks of complicity between the police and pimps leading to the prostitution of minors.

\section{KEYWORDS}

Centers of Sexual Commerce; Corruption; Sex Trafficking. 


\section{INTRODUCCIÓN}

La prostitución en México tiene un estatus cuasilegal. Desde el último tercio del siglo XIX en México ha predominado un enfoque médico y de higiene pública, que enfatiza el control, registro y chequeo sanitario obligatorio en zonas de tolerancia (Núñez Becerra 2002: 62). Las mujeres autóctonas mayores de edad con registro sanitario que trabajan en un área tolerada o en un establecimiento registrado pueden ejercer legalmente la prostitución; no así los hombres, migrantes y menores de edad (Katsulis, 2010: 126; Katsulis et al. 2010: 355), que también se prostituyen de modo ilegal en establecimientos registrados (Casillas 2008: 152).

El Reporte sobre trata del Departamento de Estado de Estados Unidos (TIP por sus siglas en inglés) ha señalado que la prostitución de menores ${ }^{1}$ ha crecido en diferentes partes de México, principalmente en áreas turísticas costeras y en las ciudades fronterizas del norte, y que muchas de las víctimas son menores procedentes de Guatemala, Honduras y EI Salvador (U.S. Department of State 2014: 271). Algunas estimaciones calculan el número de menores prostituidos en una horquilla que se extiende desde 5 mil hasta 16 mil personas (Estes et al. 2008: 130; Katsulis 2010: 139). En México el comercio sexual de menores se produce principalmente1/ en la calle; $2 /$ en sitios registrados donde se realiza el comercio sexual, y $3 /$ en lugares cuyo propósito no es el comercio sexual (Azaola 2006: 102). Los menores que ofrecen servicios sexuales en la calle constituyen el grupo más visible; pero no es el más numeroso (Azaola 2006: 103).

Este artículo estudia el comercio sexual de menores en establecimientos registrados a través de las apreciaciones de los dueños de los mismos. No analiza este fenómeno en espacios controlados por los cárteles de la droga u otro tipo de delincuentes, donde las personas son retenidas mediante el uso de la fuerza. En primer lugar, se examina la metodología utilizada. Más adelante, se analizan las categorías: explotación sexual comercial infantil y trabajo sexual juvenil. A continuación, se examina la generalización de una cultura del soborno y su impacto en el comercio sexual de menores; y, finalmente se analiza la prostitución de menores en los locales registrados en México.

\section{Metodología}

Esta investigación está sustentada en una metodología cualitativa. La técnica que se utilizó para recopilar la información fue la entrevista en profundidad y el procedimiento utilizado para seleccionar a los entrevistados fue el muestreo en cadena. ${ }^{2} \mathrm{El}$ trabajo de campo fue realizado entre 2012 y 2015 en diferentes localidades de nueve estados mexicanos: Tamaulipas, México D.F., Veracruz, Chiapas, Nuevo León, San Luis Potosí, Puebla, Tabasco y Coahuila (véase la Tabla 1).

Fueron entrevistados 73 proxenetas (47 mujeres y 26 hombres). Dos trabajaban en la calle y 71 en establecimientos cerrados (casas de citas, cantinas, night-clubs, bares, centros botaneros, salones de baile, hoteles de paso y clínicas de masajes). Sesenta y cinco establecimientos estaban registrados; el resto no tenían licencia. Más de la mitad de los establecimientos atendían a clientes de clase social baja y media-baja; un $15 \%$ atendían a clientes de clase media-baja y media, y un $30 \%$ atendían a clientes de clase media y media-alta. Los establecimientos regentados por hombres atendían sobre todo a clientes de clase media y media-alta; como contraste, los administrados por mujeres recibían principalmente a clientes de clase baja y media-baja (véase la Tabla 2).

Tabla 1.

Lugares donde se realizó el trabajo de campo.

\begin{tabular}{|l|c|c|c|c|c|c|c|c|c|}
\hline & Tamaulipas & Veracruz & México DF & Nuevo León & Chiapas & San Luis Potosí & Puebla & Coahuila & Tabasco \\
\hline $\mathbf{n}$ & 30 & 10 & 9 & 8 & 6 & 3 & 3 & 2 & 2 \\
\hline$\%$ & 41,1 & 13,7 & 12,3 & 11,0 & 8,2 & 4,1 & 4,1 & 2,7 & 2,7 \\
\hline
\end{tabular}

Fuente: Elaboración propia a partir de los datos de las entrevistas.

Tabla 2

Clase social de los clientes que acudían a los establecimientos de los entrevistados.

\begin{tabular}{|c|c|c|c|c|c|c|c|c|c|c|c|c|}
\hline & \multicolumn{2}{|c|}{ Baja } & \multicolumn{2}{|c|}{ Baja y Media-baja } & \multicolumn{2}{|c|}{ Media-baja } & \multicolumn{2}{|c|}{ Media-baja y Media } & \multicolumn{2}{|c|}{ Media } & \multicolumn{2}{|c|}{ Media y Media-alta } \\
\hline & $\mathrm{n}$ & $\%$ & $\mathbf{n}$ & $\%$ & $\mathrm{n}$ & $\%$ & $n$ & $\%$ & $\mathbf{n}$ & $\%$ & $n$ & $\%$ \\
\hline Padrotes & 5 & 19,2 & 3 & 11,5 & 3 & 11,5 & 2 & 7,7 & 6 & 23,1 & 7 & 26,9 \\
\hline Madrotas & 20 & 42,6 & 5 & 10,6 & 4 & 8,5 & 9 & 19,1 & 4 & 8,5 & 5 & 10,6 \\
\hline Total & 25 & 34,2 & 8 & 11,0 & 7 & 9,6 & 11 & 15,1 & 10 & 13,7 & 12 & 16,4 \\
\hline
\end{tabular}

Fuente: Elaboración propia a partir de los datos de las entrevistas. 
Los entrevistados tenían una edad media de 40,7 años de edad. Su experiencia en el negocio de la prostitución era elevada, ya que habían trabajado en esta actividad un promedio de 11 años, y en los establecimientos estudiados trabajaban una media de 23 mujeres (véase la Tabla 3).

La captación de los participantes se realizó a través de la utilización de sus redes sociales. Los entrevistados fueron informados sobre los objetivos generales del proyecto, sobre la institución que realizaba la investigación, y fueron invitados a participar en la misma. Aquellos que accedieron voluntariamente a la invitación fueron informados del carácter confidencial y anónimo de los datos recabados. Ninguno recibió un incentivo económico por participar, ya que esto hubiese elevado los gastos del estudio y podría haber influenciado en su consentimiento. Las entrevistas fueron conducidas con una guía que incluía tanto preguntas cerradas que recogían las características sociodemográficas y económicas de los entrevistados, como preguntas abiertas que exploraban las circunstancias que les condujeron a introducirse en el comercio sexual, las estrategias de reclutamiento utilizadas, su relación con las autoridades, etc. Todas las entrevistas fueron grabadas y transcritas. Las estrategias utilizadas para lograr la cooperación de los entrevistados fueron las siguientes: 1/ se explicó a los participantes que el propósito de la investigación era académico y el carácter anónimo de los datos. 2/ se les instruyó para que no mencionasen nombres de personas, y no se recogieron datos que revelasen su identidad ni la de otras personas aludidas. $3 /$ se redactó una guía que partía de los aspectos menos intrusivos, a los cuales los interlocutores respondían con mayor comodidad, y continuaba con aspectos cada vez más intrusivos. Además, se modificó esta guía en numerosas ocasiones para excluir cuestiones que quedaron saturadas o proporcionaban poca información, e incluir nuevos aspectos que emergieron del contacto con la realidad empírica. Finalmente, 4/ la relación discursiva con los entrevistados fue de no confrontación. Para proteger la confidencialidad de los participantes no se recogieron los nombres de los entrevistados, ni de sus establecimientos, ni datos referentes a su ubicación, y se eliminaron de las trascripciones todos los elementos que pudieran permitir identificarles.

\section{Sesgos Metodológicos Y LIMITACIONES DEL ESTUDIO}

Esta investigación presenta dos sesgos metodológicos: 1/ las entrevistas fueron realizadas en 9 de los 32 estados de la República Mexicana, y 1/ solo indaga en las apreciaciones de los proxenetas.

En primer lugar, esta investigación analiza el centro, el noreste y el sur de México; pero no los espacios donde han sido detectadas más menores prostituidos: el noroeste y las zonas turísticas y playas (U.S. Department of State 2014: 272). La inexistencia de una jurisprudencia que prohíba o tutele la prostitución ha otorgado un margen de discrecionalidad para considerarla lícita o ilícita (Torres Patiño, 2014: 3); de modo que su manejo constituye un campo de continua experimentación, y cada municipio presenta peculiaridades propias. Por ejemplo, la existencia o no de zonas de tolerancia incide en el contexto del trabajo sexual, porque donde no hay, el control sanitario es más arduo. Por lo tanto, los resultados de este estudio no representan de modo exacto la realidad de todo el país. Sin embargo, sí que ofrece datos indicativos debido a la diversidad de la geografía investigada.

En segundo lugar, esta investigación refleja de modo sesgado el comercio sexual de menores en México porque solo indaga en las apreciaciones de los padrotes y las madrotas. Es probable que los entrevistados hayan sub-reportado algunas prácticas sobre reclutamiento, empleo y trato hacia las menores. Sin embargo, estos sesgos no invalidan los resultados. La originalidad de este artículo radica en el acopio de un discurso ausente en la mayor parte de las investigaciones sobre el comercio sexual de menores. Este acercamiento ofrece nueva luz sobre este objeto de estudio y permite ahondar en la complejidad del mismo.

\section{Conceptualización de LA PROSTITUCIÓN DE MENORES}

Desde finales de los años ochenta se produce un despertar en la comunidad internacional en torno a la necesidad de combatir la prostitución de menores, la pornografía infantil y el turismo sexual. El desarrollo de nuevas tecnologías de la información y el

Tabla 3.

Características de los entrevistados.

\begin{tabular}{|l|c|c|c|c|c|c|}
\hline & Media & Moda & Mediana & Mínimo & Máximo & Desviación estándar \\
\hline Edad & 40,7 & 40 & 40 & 30 & 51 & 5,22 \\
\hline Años de experiencia como proxenetas & 11,1 & 15 & 10 & 3 & 30 & 5,16 \\
\hline $\begin{array}{l}\text { Número de mujeres que trabajan en sus } \\
\text { establecimientos }\end{array}$ & 22,9 & 20 & 20 & 9 & 100 & 12,88 \\
\hline
\end{tabular}

Fuente: Elaboración propia a partir de los datos de las entrevistas. 
crecimiento del turismo internacional fueron asociados al crecimiento del citado problema. En 1989 la Convención de Naciones Unidas sobre los Derechos del Niño subrayó la necesidad de luchar contra la explotación sexual en la prostitución y pornografía. En 1996, en el Primer Congreso Mundial contra la explotación sexual comercial de niños, celebrado en Estocolmo, se insta a los estados a criminalizar todas las formas de explotación comercial infantil, y a revisar e implementar leyes, políticas y programas para eliminar esta problemática (Alexander et al. 2000: 483). En el año 2000 se aprueba el Protocolo de Naciones Unidas para prevenir, suprimir y castigar la trata de personas, especialmente mujeres y niños, que define el "comercio sexual" de menores como "trata de personas'. Este protocolo fue incorporado en pocos años a la legislación interna de la mayor parte de los países del mundo, donde se subraya la falta de capacidad de los menores para consentir y se define como abuso sexual toda forma de comercio sexual juvenil, aunque exista consentimiento por parte del menor. Sin embargo, se asienta en un modelo de justicia criminal donde la defensa de la seguridad nacional a través de la persecución de los victimarios (la delincuencia organizada) sobresale más que la protección de las víctimas. El derecho internacional no compromete a los estados a proporcionar asistencia material, médica o de otro tipo a las víctimas (Gallagher 2010: 83). A modo de ejemplo, la evaluación que hace el Reporte TIP del esfuerzo de los países para combatir la trata subraya principalmente criterios como la participación/protección de las víctimas-testigos en procesos penales, la imposición de penas severas y el número de condenas (U.S. Department of State 2014: 272).

En el medio académico el comercio sexual de menores ha sido examinado a través de un paradigma dominante sustentado en la categoría "explotación sexual comercial infantil" (Estes et al. 2008; Casillas 2008; Reid 2011; Grace et al. 2012) y en la categoría "trabajo sexual juvenil" sostenida por un número muy reducido de académicos (Katsulis 2010; Marcus et al. 2014). La primera no encuentra diferencias entre modos forzados y no forzados, mientras que la última subraya la especificidad de los modos no forzados.

La categoría "explotación sexual comercial infantil" incluye los siguientes elementos axiomáticos: 1/ el menor es víctima de un proxeneta o traficante ${ }^{3} ; 2 /$ la prostitución de menores constituye trata de personas; 3 / el menor carece de agencia, y 4/ el menor es víctima del cliente (Estes et al. 2008; Casillas 2008; Reid 2011; Grace et al. 2012).

De acuerdo con esta categoría, proxenetas y traficantes sacan ventaja de menores, que sufrieron abuso en el hogar y padecen problemas de inseguridad económica o de drogadicción, a través de una ardua labor de reclutamiento y utilizando la seducción, la fuerza, la coerción o el engaño para introducir a jóve- nes inocentes y vulnerables en el negocio de la prostitución (Grace et al. 2012: 413; Willis y Levy 2002: 1417). El enamoramiento, las amenazas, la adicción a las drogas, la manipulación y la violencia constituyen los medios y técnicas a través de los cuales los proxenetas someten a los menores (Kennedy et al. 2007).

Algunas investigaciones realizadas en México señalan que los menores prostituidos son inducidos al consumo de drogas, reciben golpes y amenazas, o privadas de la libertad bajo un régimen de servidumbre por deudas (Estes et al. 2008: 132; Acharya 2009: 159; Esteinou 2013: 132). Otros estudios afirman que los menores son reclutados bajo falsas promesas de trabajos en bares y restaurantes, y obligadas a prostituirse (Azaola 2006: 102 y 103). También se ha señalado que las menores que huyen de sus hogares caen presas de traficantes de sexo (Reyes Parra 2007: 411). Servin et al. (2015) en un estudio cualitativo conducido en Tijuana y Ciudad Juárez sobre los factores determinantes de la entrada de menores al trabajo sexual encontraron que el $60 \%$ fueron coaccionadas; pero el $40 \%$ entraron de modo no forzado, debido a vulnerabilidades asociadas a las necesidades económicas derivadas de la maternidad adolescente. En este sentido, el concepto de violencia simbólica desarrollado por Bourdieu (2012: 3) para definir un tipo de violencia que escapa a la conciencia porque se ejerce "con la complicidad de las estructuras incorporadas que el dominado ha adquirido en la confrontación prolongada con las estructuras objetivas de dominación", puede resultar esclarecedor para entender situaciones donde el individuo que sufre este tipo de violencia, no solo no se percata, sino que es cómplice de la misma.

Pero no toda la comunidad académica comparte este punto de vista. La categoría "trabajo sexual juvenil" subraya un escenario más complejo que comporta los siguientes elementos: 1/ el menor es víctima de sus propias circunstancias; ii./ los menores en la industria de la prostitución no constituyen una categoría homogénea; iii./ la prostitución juvenil no constituye siempre trata de personas; iv./ el menor es víctima y agente autónomo, y v./ no solo el menor es víctima del cliente; sino que también el cliente puede ser víctima del menor (Katsulis 2010; Marcus et al. 2014).

La prohibición de la prostitución juvenil como constructo legal e ideológico utilizado para proteger a las personas vulnerables, es rechazada por algunos académicos que aducen que se hace caso omiso del consentimiento y condiciones laborales de los jóvenes. Se rechaza el discurso proteccionista que equipara la sexualidad de los menores con el abuso sexual, se defiende la autonomía de los jóvenes, y se subraya que la edad no constituye un criterio universal para marcar la madurez de una persona (Pheterson 2004: 135; Garaizabal 2004: 94; Agustín 2005: 110). Según Marcus et al. (2014: 241) el rol de los proxenetas en la iniciación de los menores es 
reducido, las situaciones de opresión son excepcionales, y los menores tienen cierta agencia y autonomía. Asimismo, algunos estudios realizados en el sudeste asiático no presentan a las menores como víctimas; sino que destacan la agencia de las jóvenes que se prostituyen por decisión propia (Orchard 2007: 2388; Sano 2012; Montgomery 2014 y 2015). Rende Taylor (2005: 417) explica que la tolerancia hacia la prostitución juvenil en el sudeste asiático aparece enraizada en la noción budista de la no permanencia del alma en el cuerpo físico y sus múltiples vidas en diferentes cuerpos, que contrasta con la noción judeocristiana del libre albedrío del cuerpo unido al alma en una sola vida.

En el caso de México, Katsulis (2010: 117) en un estudio sobre trabajadores sexuales juveniles en el contexto fronterizo Tijuana-California señala que el trabajo sexual constituye una estrategia de supervivencia en un entorno particular constreñido y mantenido por fuerzas macrosociales. Los menores son víctimas de constreñimientos estructurales más que de proxenetas que les obligan a prostituirse. Además, señala que las transacciones sexuales entre adultos y menores están configuradas por complejos juegos de poder y explotación tanto del adulto hacia el menor como del último hacia el primero. Asimismo, otro estudio realizado en Tijuana, donde fueron entrevistados 92 proxenetas, no reveló que estos utilizasen la fuerza o el engaño para introducir a los menores en la industria de la prostitución (Zhang 2011: 523).

\section{LA CULTURA DEL SOBORNO}

En la Constitución mexicana no existe ninguna alusión a la prostitución. La jurisprudencia de la Suprema Corte de Justicia de la Nación la ha tolerado; pero no la otorga la misma protección constitucional que a otras actividades lícitas. La prostitución es simultáneamente legal e ilícita, y quienes ejercen esta actividad son objetos de regulación pero no sujetos de derechos (Torres Patiño, 2014: 57)4. Esta falta de jurisprudencia que formalmente prohíba o tutele la prostitución ha conducido a un nivel elevado de discrecionalidad para considerarla lícita o ilícita. La ley pena lucrar con la prostitución ajena; pero permite que los dueños de algunos establecimientos con licencia ${ }^{5}$ cobijen a trabajadoras sexuales registradas que reciben tarjetas de salud (Roe-Sepowitz y Katsulis 2011: 142). La paradoja de penar y tolerar el lucro con la prostitución ajena ha conducido a la consolidación de una cultura del soborno. El pago de sobornos se ha convertido en la norma omnipresente que acatan los dueños de estos establecimientos para superar las contradicciones de un sistema que les pena y tolera al mismo tiempo. Aunque critican el pago de sobornos, los pagan puntualmente porque constituye el mecanismo que les permite operar sin contratiempos.
La Ley General de Salud (artículo 377) permite que la autoridad sanitaria requiera tarjetas de control sanitario a las personas dedicadas al comercio sexual (Torres Patiño 2014: 54). Los inspectores de salud pública municipal inspeccionan estos establecimientos para comprobar que las mujeres que trabajan allí tengan tarjetas de control sanitario y estén al corriente de los chequeos médicos semanales. Cuando una mujer carece de tarjeta sanitaria es conducida a centros de salud ${ }^{6}$ para ser revisada, y si tiene una enfermedad de transmisión sexual es llevada a Centros Ambulatorios para la Prevención y Atención en SIDA e Infecciones de Transmisión Sexual (CAPACITS). Los inspectores de salud son escoltados por policías municipales cuando existe riesgo de violencia ( $v g r$. los fines de semana). Si una mujer que no tiene tarjeta sanitaria rehúsa a acompañarles al centro de salud o si una mujer que tiene una enfermedad de transmisión sexual sigue trabajando, los inspectores de salud reportan la situación a los policías municipales para que intervengan.

La Policía Municipal Preventiva realiza operativos en establecimientos mercantiles y en la vía pública en respuesta a quejas ciudadanas (Torres Patiño 2014: 53); pero los funcionarios se rigen por sus propios criterios, lo que favorece la corrupción (Casillas 2008: 152). En los establecimientos registrados su función ${ }^{7}$ consiste en realizar visitas domiciliarias para supervisar el cumplimiento de horarios o constatar si se violó el tipo de licencia otorgada, además de detectar si se cometió algún acto ilícito: empleo de menores, trasiego de drogas, adulteración de bebidas, etc. Ellos no tienen competencias para realizar inspecciones de salud o migratorias. Sin embargo, la policía escruta principalmente a los clientes (Katsulis et al. 2010: 356). Las autoridades estacionan sus vehículos al lado de estos establecimientos para registrar a los clientes y mujeres que salen de los mismos. Estos operativos no se realizan al azar; tienen como objetivo consolidar la cultura del soborno y hacer comprender a quienes no pagan que no podrán operar sus negocios de modo rentable. Aquellos que no pagan cuotas son acosados; pero quienes pagan sobornos no son molestados (Reyes Parra 2007: 148). Como señalaba un padrote veracruzano de 45 años de edad: "me piden dinero para dejarme trabajar bien tranquilo y no registrar la cantina, les he tenido que pagar para que me dejen trabajar; cuando no lo hago vieras que se la pasan en la esquina o vuelta y vuelta mirando que salgan los clientes, o las mujeres, y los revisan, piden registros y los molestan, cuando eso pasa los clientes ni vienen por miedo a la misma policía". Como los clientes buscan el anonimato y huyen de la presencia de la policía, los dueños de los locales deben llegar a acuerdos con las autoridades para que no se acerquen a sus establecimientos.

Finalmente, el Instituto Nacional de Migración o la Policía Federal realizan inspecciones para detectar la presencia de mujeres que no pueden acreditar 
su estancia legal en México. Los agentes migratorios realizan solos las inspecciones cuando no hay indicios de violencia; pero como no llevan armas frecuentemente participan en operativos conjuntos con inspectores de salud y policías municipales. Los agentes migratorios sancionan a las mujeres foráneas por realizar una actividad económica sin documentos; pero no sancionan a los dueños de los establecimientos.

En este estudio no se registró el pago de sobornos a inspectores de salud pública municipal. Esto puede obedecer a que el control sanitario favorece los intereses de los dueños de estos establecimientos. Muchos clientes demandan que las mujeres estén al corriente de los controles de salud, y las visitas de los inspectores de salud tienen como objeto obligar a las mujeres a seguir estos controles. Ellos no sancionan a los dueños. Por el contrario, el pago de sobornos a policías y agentes migratorios constituía una norma no escrita. Los sobornos pagados a los policías municipales mostraban una regularidad; sin embargo, los pagados a los agentes migratorios presentaban un carácter irregular y su cuantía era menor. Esto obedece a que las inspecciones policiales pueden conducir al cierre del local o a procesos penales; mientras que las inspecciones de migración solo conducen a una pérdida de trabajadoras. Como consecuencia, solo están dispuestos a hacer pequeños pagos a los agentes migratorios. Como decía un padrote veracruzano de 50 años de edad "a veces les doy un poco; pero ellos con poco se conforman".

Casi todos los establecimientos analizados pagaban algún tipo de soborno a los policías municipales. Una quinta parte de los entrevistados no especificaron que pagasen sobornos a las autoridades policia- les (véase la Tabla 4). El impago de sobornos a la policía es más frecuente en áreas donde el poder de los cárteles de la droga contrasta con la debilidad de las autoridades. En estos casos los dueños de los locales pagan sobornos a los delincuentes; pero no a la policía. Aunque en otras zonas el pago de sobornos a la delincuencia organizada no exime a los patrones de los pagos a las autoridades policiales.

Otra quinta parte de los entrevistados señalaron que pagaban un soborno a la policía; pero no especificaron la cuantía. Algunos dijeron que los pagos no los hacían de modo regular y no siempre pagaban lo mismo. Como contraste, casi un $60 \%$ admitieron que pagaban un soborno fijo de manera periódica. Estos pagos generalmente se realizaban una vez al mes; pero en ocasiones eran semanales. Los entrevistados pagaban una media de 7063 pesos mensuales. Los padrotes pagaban cantidades más elevadas que las madrotas porque los establecimientos de los primeros generaban mayores ganancias (véase la Tabla 5).

Asimismo, en la Tabla 6 se aprecia que el pago de sobornos para emplear mujeres centroamericanas que no pueden acreditar su presencia legal en México está muy extendido.

El pago de sobornos no únicamente sirve para proteger el anonimato de los clientes; en ocasiones es utilizado para fomentar el comercio sexual juvenil. Algunos proxenetas se arriesgan a dar empleo a menores porque son más demandadas por los clientes (Acharya 2009: 154). Ellos reciben un porcentaje de la ganancia generada por las mujeres, que se puede situar entre el $25 \%$ y el $50 \%$. Cuando emplean a menores de edad este porcentaje se eleva porque se exponen a severas penas carcelarias. Este elemen-

Tabla 4.

Sobornos pagados a los policías municipales por los entrevistados.

\begin{tabular}{|l|c|c|c|c|c|c|}
\hline \multirow{2}{*}{} & \multicolumn{2}{|c|}{ Padrotes } & \multicolumn{2}{|c|}{ Madrotas } & \multicolumn{2}{|c|}{ Total } \\
\cline { 2 - 7 } & $\mathbf{n}$ & $\mathbf{\%}$ & $\mathbf{n}$ & $\mathbf{\%}$ & $\mathbf{n}$ & $\%$ \\
\hline No especificó que pagase sobornos. & 7 & 26,9 & 8 & 17,0 & 15 & 20,5 \\
\hline Pagaba un soborno a la policía; pero no especificó cuánto. & 1 & 3,9 & 14 & 29,8 & 15 & 20,5 \\
\hline Pagaba una cuota regular a la policía y especificó cuánto. & 18 & 69,2 & 25 & 53,2 & 43 & 58,9 \\
\hline Total. & 26 & 100 & 47 & 100 & 73 & 100 \\
\hline
\end{tabular}

Fuente: Elaboración propia.

Tabla 5.

Sobornos pagados mensualmente a la policía por los entrevistados (pesos mexicanos) ( $n=43)$.

\begin{tabular}{|l|c|c|c|c|c|c|c|}
\hline & $\mathbf{n}$ & Media & Moda & Mediana & Mínimo & Máximo & Desviación estándar \\
\hline Padrotes & 18 & 8167 & 10000 & 9500 & 1000 & 13000 & 3276 \\
\hline Madrotas & 25 & 6268 & 10000 & 6000 & 500 & 10000 & 3220 \\
\hline Total & 43 & 7063 & 10000 & 8000 & 500 & 13000 & 3737 \\
\hline
\end{tabular}

Fuente: Elaboración propia a partir de los datos de las entrevistas. 
Tabla 6.

Sobornos pagados por los entrevistados que emplean a mujeres centroamericanas.

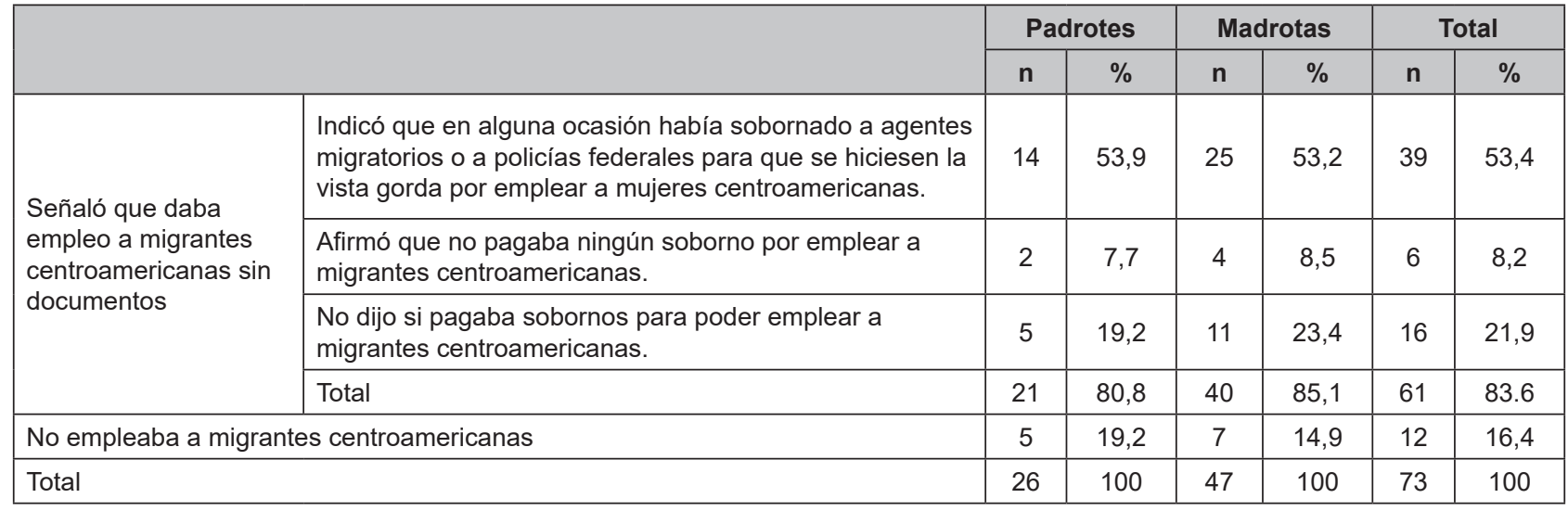

Fuente: Elaboración propia a partir de los datos de las entrevistas.

to desincentiva a muchos patrones; pero para otros es un aliciente. Una menor es demandada por más clientes, recibe cantidades más elevadas por cada servicio realizado, y su patrón la descuenta un porcentaje más elevado de sus ganancias.

La relación cimentada con las autoridades a través del pago de sobornos, en ocasiones sirve para construir un halo de protección (Reyes Parra 2007: 419; U.S. Department of State 2014: 272). Algunos se arriesgan a dar empleo a menores, bien porque las autoridades se hacen la vista gorda cuando implementan algún operativo, o bien, porque son avisados antes de que se implementen estos operativos. Esto no significa que el pago de sobornos implique una protección automática ante el empleo de menores de edad. Según los entrevistados, el trasiego de drogas o el empleo de mujeres foráneas son aspectos más fáciles de arreglar mediante el pago de sobornos que el empleo de menores.

\section{EL EMPLEO DE MENORES DE EDAD EN LOS LOCALES DE COMERCIO SEXUAL EN MÉXICO}

Cuando la edad de las mujeres avanza, el número de establecimientos de comercio sexual donde son admitidas se estrecha (Acharya 2009: 151; 2013: 116; Esteinou 2013: 131). En los establecimientos examinados las jóvenes eran admitidas desde edades iniciales comprendidas entre 13 y 25 años hasta edades máximas que oscilaban entre los 21 y los
50 años de edad (véase la Tabla 7). La fluctuación entre las edades máxima y mínima de entrada en estos establecimientos aparecía relacionada con el nivel económico de los clientes que acudían a los mismos. Los clientes de clases bajas no pueden elegir, buscan servicios baratos. Por lo tanto, los establecimientos que atienden a una clientela de bajos recursos no suelen establecer un límite máximo de edad para poder trabajar allí. Aquí trabajan mujeres de hasta cincuenta años de edad. Como contraste, aquellos establecimientos que atienden a una clientela de más recursos no suelen admitir a mujeres de más de 30 años de edad. Una mujer que tenía una cantina que atendía a clientes de clase social baja decía que ella empleaba a mujeres de más de 25 años de edad; pero cuando llegaban a su establecimiento mujeres de 16 a 25 años las conducía con un padrote que atendía a una clientela más selecta. Ella pensaba que en su cantina mujeres tan jóvenes perderían la oportunidad de ganar más dinero.

\footnotetext{
"si me llegan de 16 a 25 o 23 las paso al padrote que me pide mujeres, él las acomoda a trabajar en otros lugares donde ellas sacan más dinero" (Madrota veracruzana de 45 años de edad).
}

Para comprobar la veracidad de las respuestas de los entrevistados respecto al empleo de menores de edad, este aspecto fue indagado en diferentes momentos de la interacción conversacional con objeto de observar si se producían inconsistencias en las respuestas. Un $20 \%$ de los entrevistados señalaron en un primer momento que únicamente empleaban a mujeres mayores de 18 años de edad; pero, cuando

Tabla 7.

Edad mínima y máxima de las mujeres admitidas por los entrevistados $(n=73)$.

\begin{tabular}{|l|c|c|c|c|c|c|}
\hline & Media & Moda & Mediana & Mínimo & Máximo & Desviación estándar \\
\hline Mínima & 17.6 & 18 & 18 & 13 & 25 & 1,59 \\
\hline Máxima & 36.7 & 50 & 35 & 21 & 50 & 9,32 \\
\hline
\end{tabular}

Fuente: Elaboración propia. 
avanzó la entrevista revelaron que ocasionalmente empleaban a menores o las conducían con otros proxenetas. Aquellos entrevistados que ofrecieron respuestas consistentes sobre el empleo de menores aparecen definidos en la Tabla 8 como "establecimientos que emplean a menores regularmente". Quienes respondieron de modo consistente que nunca daban empleo a menores aparecen definidos como "establecimientos que no emplean a menores de edad". Donde encontramos inconsistencias se utilizó el término "emplean menores de modo ocasional".

\section{Los establecimientos que regularmente emplean a menores}

Un $29 \%$ de los entrevistados afirmaron que en sus locales había jóvenes de menos de 18 años (véase la Tabla 8); pero señalaban que no utilizaban el engaño o la coacción, sino que las menores buscaban trabajo en estos establecimientos debido a circunstancias económicas. Generalmente eran madres solteras que necesitaban recursos para proporcionar un techo y alimentar a sus hijos.

Los dueños de estos establecimientos no siempre dan empleo a las menores; en algunos casos porque son demasiado jóvenes, en otros porque carecen de experiencia, y otros patrones solo admiten a quienes presentan determinadas características familiares (Izcara Palacios 2017: 41).
El empleo de menores obedece a la preferencia de los clientes. Los clientes que gastan más dinero, acuden a establecimientos donde las mujeres son más jóvenes y esbeltas. Como decía un padrote del Estado de México de 42 años de edad: "aquí llegan a trabajar desde menores, desde los 15 años hasta los 25 o 26; yo les pido que estén jóvenes, eso porque así los clientes las prefieren mejor y son más pagadas". Aunque, la demanda de niñas de corta edad es inusual. Como aparece reflejado en las siguientes citas, algunos entrevistados señalaban que no empleaban a jóvenes de menos de 15 años de edad porque los clientes no demandaban niñas de tan corta edad.

"vienen desde 15 años para arriba; tengo clientes que las quieren y ellas vienen porque necesitan" (Madrota tamaulipeca de 42 años de edad).

"menor de 15 años no les doy trabajo porque así no las quieren, ya de 15 sí las quieren" (Madrota chiapaneca de 40 años de edad).

Tres son las características que buscan los proxenetas en las menores a quienes prostituyen en sus establecimientos: i./ la ausencia de lazos sociales (Azaola 2006: 103); ii./ la experiencia, y iii./ la posesión de una carga familiar.

La ausencia de lazos familiares es un elemento que protege a los proxenetas. El artículo 13 de la ley de trata (Reforma del 19-03-2014) impone penas de

Tabla 8.

El empleo de menores de edad en los establecimientos estudiados.

\begin{tabular}{|c|c|c|c|}
\hline Tipo & Causa del empleo de menores & $\mathbf{n}$ & $\%$ \\
\hline \multirow{7}{*}{$\begin{array}{l}\text { Emplean a menores } \\
\text { regularmente }\end{array}$} & Emplean a menores porque las demandan los clientes & 14 & \multirow{7}{*}{28,8} \\
\hline & Solo emplean a mayores de 15 años & 2 & \\
\hline & $\begin{array}{l}\text { Aceptan a menores sin experiencia; aunque las advierten que si quedan embarazadas las } \\
\text { despedirán }\end{array}$ & 2 & \\
\hline & $\begin{array}{l}\text { Solo da trabajo a menores con hijos; pero no trabajan en el establecimiento. Las contactan } \\
\text { por teléfono }\end{array}$ & 1 & \\
\hline & Solo acepta menores con hijos que se han despegado de sus familiares & 1 & \\
\hline & Admite a todas las menores & 1 & \\
\hline & Total & 21 & \\
\hline \multirow{6}{*}{$\begin{array}{l}\text { Emplean menores } \\
\text { de modo ocasional }\end{array}$} & Dan empleo a menores; pero no están en el establecimiento. Son contactadas por teléfono & 4 & \multirow{6}{*}{20,5} \\
\hline & Emplea a menores a quienes proporcionan un documento de identidad falso & 5 & \\
\hline & Emplea a menores que aparentan tener más edad & 3 & \\
\hline & No da empleo a menores en su establecimiento; pero recluta menores para otro proxeneta & 2 & \\
\hline & Emplea a menores que tienen hijos & 1 & \\
\hline & Total & 15 & \\
\hline \multirow{4}{*}{$\begin{array}{l}\text { No emplean a } \\
\text { menores }\end{array}$} & No emplea a menores por temor a la ley & 27 & \multirow{4}{*}{49,3} \\
\hline & $\begin{array}{l}\text { No emplea a menores porque carecen de experiencia y son incapaces de satisfacer a los } \\
\text { clientes }\end{array}$ & 6 & \\
\hline & Están en contra del empleo de menores. & 3 & \\
\hline & Total & 36 & \\
\hline \multicolumn{2}{|c|}{ No habló de este tema en la entrevista } & 1 & 1,4 \\
\hline \multicolumn{2}{|l|}{ Total } & 73 & 100 \\
\hline
\end{tabular}

Fuente: Elaboración propia a partir de los datos de las entrevistas. 
hasta 30 años de prisión a quien se beneficie de la explotación de personas menores de edad a través de la prostitución. Un proxeneta que emplea a menores puede ser descubierto en un operativo policial o a través de una denuncia realizada por familiares. Mediante el pago de sobornos los patrones pueden evitar que las autoridades realicen indagaciones en sus establecimientos, y dando empleo a menores sin lazos familiares u otro tipo de capital social minimizan el riesgo de ser denunciados. Una joven con fuertes vínculos familiares puede denunciar a su patrón apoyada por su familia, o los familiares pueden denunciar al proxeneta para alejar a la menor del mundo de la prostitución. Por el contrario, una menor sin vínculos familiares es más vulnerable; es poco probable que denuncie a la persona de quien depende para sobrevivir. Como señalaba una madrota tamaulipeca de 42 años de edad: "a veces sí las acepto (menores de edad), pero les digo que no quiero problemas con sus familiares; pero por lo regular son mujeres que ya tienen hijos y por eso vienen a trabajar aquí a la cantina".

La experiencia constituye otro requisito importante en los establecimientos registrados, ya que la mayor parte de los clientes no demandan jóvenes inexperimentadas. Esto no significa que los proxenetas no empleen a jóvenes sin experiencia. Aquellos entrevistados que admitieron haber dado empleo a niñas sin experiencia afirmaron que lo hicieron debido a la insistencia de las primeras. Los entrevistados, lejos de buscar jóvenes inexpertas, mostraban cierta reticencia a darles empleo. Ellas son quienes pueden acarrearles mayores problemas, porque es más fácil que queden embarazadas, que se arrepientan de su decisión o que sean reclamadas por algún familiar. Un padrote y una madrota afirmaban que advertían a las menores sin experiencia que debían utilizar algún método anticonceptivo, ya que si quedaban embarazadas las expulsarían de sus locales.

\footnotetext{
"Sí las acepto (menores sin experiencia), pero les digo que no quiero problemas con nadie y que se cuiden de un embarazo porque después no hay quien responda" (Padrote tamaulipeco de 45 años de edad).

"yo lo que hago es que se cuiden de un embarazo o una enfermedad, por eso les pido que con los clientes usen condón y que se inyecten para no embarazarse, ya embarazadas no hay trabajo para ellas" (Madrota veracruzana de 41 años de edad).
}

Servin et al. (2015) han señalado que el embarazo adolescente constituye el común denominador asociado a la percepción del trabajo sexual como una respuesta a sus necesidades económicas. Asimismo, la posesión de una carga familiar es otra de las características que buscan los proxenetas en las menores. Una menor sin cargas familiares puede cambiar de opinión y denunciar a su patrón; pero no así una que debe mantener a un hijo, ya que la última necesita más recursos económicos que la primera. Una de las entrevistadas decía que solo daba trabajo a menores con hijos. Aunque estas menores no trabajaban dentro de su local. Ella tenía clientes fijos que buscaban menores de edad. Cuando estos clientes pedían un servicio, telefoneaba a las menores y concertaba una cita entre el cliente y la menor.

\begin{abstract}
"menores de edad no pueden estar aquí; sí trabajo con menores de edad, pero por teléfono, y solo acepto a las que tienen necesidades, que tienen hijos, por ello les doy clientes, para que ayuden a sus hijos" (Madrota veracruzana de 40 años de edad).
\end{abstract}

Finalmente, un proxeneta que trabajaba en la caIle afirmaba que empleaba a todo tipo de mujeres sin importar la edad. Él no tenía miedo a ser detenido por las autoridades policiacas porque decía que tenía contactos que le protegían.

\section{Los establecimientos que emplean a menores de modo ocasional}

Una quinta parte de los entrevistados manifestaron en un primer momento que solo empleaban a mayores de edad; pero a medida que avanzó la interacción conversacional admitieron que en ocasiones sí que empleaban a menores (véase la Tabla 8). Los patrones que reconocían abiertamente que empleaban a menores de edad no solían temer a las autoridades, ya que tenían algún tipo de protección. Como decía un padrote del Estado de México de 42 años de edad: "les pago a esas personas (autoridades) para que nos dejen trabajar; cuando hay redadas yo sé y no las dejo trabajar". Sin embargo, los que terminaron admitiendo que empleaban menores de modo ocasional no solían estar protegidos; por lo tanto, debían ser más precavidos.

Los patrones que no tienen arreglos que les protegen no suelen permitir que las menores trabajen en sus establecimientos. Cuando un cliente demanda una menor, la telefonean y la ponen en contacto con él. Una madrota tamaulipeca de 42 años de edad decía que cuando llega una menor "no la dejo trabajar a simple vista; pero si le interesa mucho, me deja sus datos, y cuando llega un cliente que la solicite, les llamo, todo a escondidas por las autoridades, que sí cuidan mucho esto de la prostitución en menores de edad". Otras veces, los dueños de algunos establecimientos reclutan jóvenes para proxenetas que emplean a menores.

Otros patrones utilizan dos estrategias para que las autoridades no detecten que alguna de las mujeres que se encuentran en sus establecimientos es menor de edad: i./ les proporcionan documentos apócrifos, o ii./ dan empleo a jóvenes que aparentan tener más edad.

El uso de documentos apócrifos ha sido constatado en numerosas investigaciones realizadas en México (Orozco y Hernández 2011: 49 y 123; Mon- 
tiel Torres 2011: 13; Reyes Parra 2007: 117). Esta es una estrategia muy efectiva, ya que la menor puede engañar a las autoridades mostrando una credencial de otra persona con algún parecido físico. Un padrote de México DF de 39 años de edad manifestó que había recurrido al empleo de documentación apócrifa en una ocasión:

"Ella es chica y le di trabajo porque tiene 2 niños chicos y batalla mucho [...] Yo le ayudé a conseguir una credencial de elector de otra persona mayor de edad. Así, si llegan a venir a preguntar por ella, ella misma les enseña la credencial".

Pero, otros señalaron que hacían uso de esta estrategia de modo más recurrente. A estas jóvenes les cambian su apariencia con maquillaje, haciendo que aparenten más edad para que no despierten sospechas.

"[Cuando llega una menor] le consigo una credencial que diga que es mayor de edad por aquello que lleguen a revisar la cantina no la encuentren como menor de edad. Hay mujeres que llegan con 16 años y tenemos que decir que tienen 18 años o más" ( $\mathrm{Pa}$ drote tamaulipeco de 50 años de edad).

"Las dejo que trabajen, pero con cuidado, con credenciales de mujeres mayores para que no las molesten por su edad" (Madrota de Tabasco de 46 años de edad).

Las autoridades también pueden ser engañadas por menores que aparentan tener más edad. A diferencia de las menores que son maquilladas para modificar su apariencia, las jóvenes cuya fisonomía hace que aparenten más edad de la que tienen no despiertan tanto interés en los clientes. Los patrones las emplean porque están próximas a la mayoría de edad o porque fueron engañados. Aunque, no suelen despedir a una joven que mintió sobre su edad.

"Hay unas que son menores de edad y representan más edad; ellas a veces se quedan a trabajar" (Madrota guerrerense de 41 años de edad).

"Algunas menores de edad tienen la cara de más edad y a ellas sí las dejo trabajar porque me dicen que tienen más años, con los días me doy cuenta que son menores" (Madrota chiapaneca de 37 años de edad).

"Yo les pido que tengan más de 18 años o que estén por cumplirlos" (Madrota salvadoreña de 41 años de edad).

\section{Los establecimientos que no emplean a menores de edad}

La mitad de los entrevistados respondieron que en sus establecimientos no empleaban a menores de edad. Ellos se manifestaban en contra de las leyes que prohibían la prostitución de menores y afirmaban que la ley debía de contemplar excepciones, como las jóvenes con cargas familiares. Sin embargo, de- cían que no les daban empleo porque el riesgo sobrepasaba los beneficios que obtendrían.

Veintisiete entrevistados deseaban dar empleo a las menores que llegaban a sus establecimientos en busca de trabajo; pero, no las admitían para no tener problemas con las autoridades.

"cuando llega una chiquita de edad no la dejo trabajar porque si se queda a trabajar me da problemas y me va mal" (Padrote chiapaneco de 40 años de edad).

"cuando vienen chiquitas de edad no las dejo trabajar porque después a mí me va mal en el trabajo, aquí me pueden clausurar por ellas, por eso no les doy trabajo" (Madrota tamaulipeca de 35 años de edad).

"cuando llega una menor de edad no le doy trabajo porque no quiero llegar a tener problemas". (Madrota de México DF de 31 años de edad).

No todos los patrones que no permiten que en sus establecimientos trabajen menores lo hacen por temor a la ley. Seis entrevistados respondieron que no daban empleo a menores de edad porque carecían de experiencia para satisfacer a los clientes. Los clientes que acudían a estos locales demandaban mujeres experimentadas, que conociesen trucos y asumiesen un rol activo. Por el contrario, el rol más pasivo de las menores sin experiencia no era del agrado de los clientes.

"Cuando llegan a ser menores de edad no las quiero porque no tienen experiencia, y eso es lo que se requiere aquí" (Padrote tamaulipeco de 50 años de edad).

"si es menor de edad no se le deja trabajar, eso porque no conviene que ellas trabajen, no llegan a tener experiencia y no tienen una madurez" (Padrote veracruzano de 45 años de edad).

"cuando son menores de edad no tienen la experiencia que los hombres quieren y yo aquí no les doy trabajo. No las dejo trabajar porque no son buenas y no me sacan el trabajo; no hacen las cosas bien como las hacen las que ya saben trabajar" (Madrota de Puebla de 42 años de edad).

Por otra parte, tres madrotas se manifestaban en contra de la prostitución juvenil, porque pensaban que las menores de edad carecían de madurez para realizar este trabajo. Ellas pensaban que empleándolas ganarían más, porque los clientes preferían a las más jóvenes; pero creían que el oficio de la prostitución requería de una madurez que no tenían las menores. Esto aparecía reflejado en expresiones como: "yo no acepto a menores porque en ellas veo como si fueran mis hijas" (Madrota tamaulipeca de 35 años de edad); "son muy chicas y no tienen la madurez en su cabeza" (Madrota tamaulipeca de 45 años de edad), o "no las dejo trabajar porque creo que las menores no están listas para ejercer la prostitución" (Madrota guerrerense de 35 años de edad). 


\section{CONCLUSIÓN}

La prostitución de menores es frecuente en centros de comercio sexual registrados; pero permanece velada porque, como se desprende de esta investigación, muchas menores son escondidas o disfrazadas por los dueños de establecimientos dedicados a la prostitución. Además, la generalización de una cultura del soborno conduce a la solidificación de redes de complicidad entre autoridades y proxenetas, que en ocasiones son aprovechadas por los últimos para emplear a menores sin riesgo.

Los entrevistados dibujaban un inverosímil escenario donde ellos se describían como personas altruistas que se arriesgaban para proporcionar un modo de subsistencia a jóvenes que no tenían nada. Aunque algunas madrotas sí mostraban un interés genuino por el bienestar de las menores. Ellas fueron víctimas de explotación sexual y rehusaban admitir a menores. Sin embargo, como ha sido señalado en otros estudios (Orchard 2007; Katsulis 2010; Zhang 2011; Sano 2012; Marcus et al. 2014; Montgomery 2014 y 2015) resulta creíble la idea de que algunas menores constreñidas por una combinación de vulnerabilidades puedan ser a la vez víctimas y agentes autónomos que buscan empleos en establecimientos de comercio sexual.

La lucha contra la explotación sexual de menores se ha enraizado en un modelo de justicia criminal que mide sus logros por el número de aprehensiones más que por la atención a las víctimas. Este modelo reduce la etiología de la prostitución de menores a un asunto de gente mala que hace cosas malas a gente buena, y crea la falsa ilusión de que puede eliminarse sin modificar las estructuras socioeconómicas que la nutren. ${ }^{8}$ La explotación sexual de menores es un fenómeno poliédrico. La forzada obedece a prácticas activas de reclutamiento donde la víctima es retenida con amenazas y violencia. La no forzada obedece a constreñimientos que llevan a la menor a buscar en el trabajo sexual una salida a sus problemas económicos sin que los proxenetas tengan que desplegar prácticas activas de reclutamiento. La primera puede frenarse encarcelando a quienes las explotan; pero el modelo de justicia criminal es poco eficaz para combatir los casos donde las menores perciben el comercio sexual como una opción factible. La pros- titución no forzada de menores no puede definirse como prostitución voluntaria, porque los proxenetas se benefician de la vulnerabilidad de las jóvenes (Servin et al. 2015). Pero las penas impuestas a los proxenetas que emplean modos no forzados deben ser menores a las impuestas a quienes utilizan la violencia y la coacción. Además, la corrupción no permite implementar adecuadamente el modelo de justicia criminal. Aquellos que tienen mayor capacidad para corromper a la autoridad (vgr. los cárteles de la droga) son quienes utilizan métodos más violentos, y quienes más probablemente queden impunes. Es probable que el modelo de justicia criminal persiga los casos donde resulta menos eficaz el combate y no ataque aquellas situaciones donde es más necesario. Por lo tanto, se debe combatir la corrupción policial y la cultura del pago de sobornos.

Las menores prostituidas de modo forzado es menos probable que vuelvan al comercio sexual si quienes las explotan son encarcelados; pero es muy probable que las no forzadas sí retornen porque el modelo de justicia criminal no resuelve los factores estructurales que hicieron que encontrasen en esta industria una opción viable. La pobreza y la desigualdad social forman un caldo de cultivo que alimenta la prostitución juvenil. Por lo tanto, deben diseñarse programas que protejan a menores que padezcan situaciones socio-económicas tan precarias que perciban el comercio sexual como una solución. La lucha contra la prostitución de menores debe concentrar el grueso de los recursos humanos y económicos en la atención a las víctimas. Posiblemente las jóvenes que ingresaron de modo no forzado al comercio sexual necesiten mayor atención porque es menos probable que cuenten con un entorno familiar que las arrope. Sin embargo, numerosos estudios (Mitchell et al. 2010; Halter 2010, Fichtelman 2014; U.S. Department of State 2014: 273) indican que mientras las menores forzadas reciben atención, las no forzadas son criminalizadas y tratadas como no merecedoras.

\section{Agradecimientos}

Fuente de financiación: Esta investigación ha sido financiada por SEP/CONACYT. Proyecto $\mathrm{N}^{\circ}$ CB-2013-01 220663 titulado "Trata y prostitución en México".

\section{NOTAS}

1. En este artículo establezco una distinción entre el término "trabajo sexual juvenil" para definir la entrada temprana (antes de cumplir los 18 años) al comercio sexual de manera no forzada, y los vocablos "explotación sexual comercial infantil", "explotación sexual de menores", o "menores prostituidas" para definir la entrada forzada de menores de edad al comercio sexual. Desde el punto de vista legal esta distinción no es relevante; pero, estos términos designan situaciones distintas. Cuando utilizo los términos "prostitución de menores" o "comercio sexual de menores" reflejo

ambas situaciones: la prostitución forzada y la no forzada de menores.

2. El diseño metodológico fue aprobado por el Consejo Nacional de Ciencia y Tecnología de México (CONACYT).

3. Persona que facilita el transporte de la menor.

4. Una excepción es la sentencia emitida por el Juzgado Primero de Distrito en Materia Administrativa en el Distrito Federal el 31 de enero de 2014. Esta sentencia 
señala que la prostitución está amparada por el artículo 5 de la Constitución, introduce la categoría del trabajo sexual y reconoce a quienes ejercen la prostitución como sujetos de derechos (Torres Patiño 2014: $61)$.

5. Esta licencia, que debe renovarse anualmente, es otorgada por la Dirección de Recaudación de la Secretaría de Finanzas a algunos establecimientos (cabarets, centros nocturnos, bares, cantinas, tabernas, etc.) que se encuentran ubicados a más de 200 metros de distancia de planteles educativos, centros culturales, hospitales, sanatorios, centros, asilos, centros asistenciales, centros donde trabajan más de 100 empleados, templos religiosos, centros de convivencia o instalaciones deportivas, edificios públicos o de asistencia social, y no se encuentre ubicado en zona residencial. Además, la autoridad sanitaria debe acreditar que el local reúne los requisitos sanitarios en vigor. La licencia permite realizar una actividad lícita: vender bebidas alcohólicas; pero se tolera el ejercicio de la prostitución.

\section{REFERENCIAS BibLIOGRÁfICAS}

Acharya, A. K. 2009. Una nueva forma de esclavitud humana. El tráfico de mujeres en México. Monterrey: Universidad Autónoma de Nuevo León.

Acharya, A. K. 2013. "Trafficking of women in Mexico: Sexual exploitation and reproductive health status". Pp. 109122 en Global Perspectives on Prostitution and Sex Trafficking, editado por R. L. Dalla, L. M. Baker, J. Defrain y C. Williamson. Plymouth: Lexington Books.

Agustín, L. M. 2005. "La industria del sexo, los migrantes y la familia europea". Cuadernos Pagu 25: 107-128. https://doi.org/10.1590/S0104-83332005000200005

Alexander, S., S. Meuwese y A. Wolthuis. 2000. "Policies and developments relating to the sexual exploitation of children: The legacy of the Stockholm Conference". European Journal on Criminal Policy and Research 8: 479501. https://doi.org/10.1023/A:1008759107302

Azaola, E. 2006. "The sexual exploitation of children in Mexico". Police Practice and Research 7: 97-110. https:// doi.org/10.1080/15614260600676742

Bourdieu, P. 2012. "Symbolic violence". Revista Latina de Sociología 2: 1-4.

Casillas, R. 2008. "Abuso y explotación sexual de niñas, adolescentes y mujeres en la Ciudad de México", Pp. 151183 en Explotación sexual comercial infantil. Un manual con perspectiva de género y derechos humanos, compilado por R. Pastor Escobar y R. Alonso Nogueira. México DF: UBIJUS Editorial.

Esteinou, R. 2013. "Selling bodies and sexual exploitation: Prostitution in Mexico". Pp. 123-140 en Global Perspectives on Prostitution and Sex Trafficking, editado por R. L. Dalla, L. M. Baker, J. Defrain y C. Williamson. Plymouth: Lexington Books.

Estes, R. J., A. Gauthier y E. Azaola. 2008. "La explotación sexual comercial de niños en la región de América del Norte". Pp. 107-149 en Explotación sexual comercial infantil. Un manual con perspectiva de género y derechos humanos, compilado por R. Pastor Escobar y R. Alonso Nogueira. México DF: UBIJUS Editorial.

Fichtelman, E. B. 2014. "The Double Entendre of Juvenile Prostitution: Victim versus Delinquent and the Necessi-
6. Por ejemplo, en Tamaulipas se denominan Centros de Atención Integral y Reducción de Riesgos Sexuales (CAIRRS).

7. Esta función le corresponde a la Secretaría de Finanzas; pero está transferida a funcionarios (policías municipales) designados por los presidentes municipales. Estas visitas domiciliarias pueden resultar en la cancelación de la licencia de funcionamiento (además de sanciones de carácter penal correspondiente) en casos de alteración del orden, venta de bebidas alcohólicas a menores, realización de actos constitutivos de delitos, etc.

8. Como resultado, se prioriza la creación de refugios especializados para víctimas de trata que participan en procesos penales, como el de alta seguridad de México DF, donde la víctima es re-victimizada para proteger la seguridad del Estado. Como señala el Reporte TIP, las víctimas que no colaboran en los procesos corren el riesgo de ser victimizadas, como el caso de una menor hondureña sentenciada a 27 años de cárcel en Chiapas (U.S. Department of State 2014: 273).

ty of State Uniformity". Juvenile \& Family Court Journal 65: 27-46. https://doi.org/10.1111/jfcj.12021

Gallagher, A. T. 2010. The International Law of Human Trafficking. New York: Cambridge University Press. https:// doi.org/10.1017/CBO9780511761065

Garaizábal, C. 2004. "ONG y derechos humanos: la experiencia de Hetaira". Pp. 85-95 en Trabajadoras del sexo. Derechos, migraciones y tráfico en el siglo XXI, editado por R. Osborne. Barcelona: Edicions Bellaterra.

Grace, L. G., M. Starck, J. Potenza, P. A. Kenney y A. H. Sheetz. 2012. "Commercial Sexual Exploitation of Children and the School Nurse". Journal of School Nursing 28: 410417. https://doi.org/10.1177/1059840512448402

Halter, S. 2010. "Factors That Influence Police Conceptualizations of Girls Involved in Prostitution in Six U.S. Cities: Child Sexual Exploitation Victims or Delinquents?". Child Maltreatment 15: 152-160. https://doi. org/10.1177/1077559509355315

Izcara Palacios, S.P. 2017. "Prostitution and migrant Smuggling Networks Operating between Central America, Mexico and the United States". Latin American Perspectives 44: 31-49. https://doi.org/10.1177/0094582X17699910

Katsulis, Y. 2010. "Youth sex workers on the US-Mexico Border". Wagadu: A Journal of Transnational Women's \& Gender Studies 8: 115-143.

Katsulis, Y., V. Lopez, A. Durfee y A. Robillard. 2010. "Female sex workers and the social context of workplace violence in Tijuana, Mexico". Medical Anthropology Quarterly 24: 344362. https://doi.org/10.1111/j.1548-1387.2010.01108.x

Kennedy, M. A., C. Klein, J. T. Bristowe, B. S. Cooper y J. C. Yuille. 2007. "Routes of recruitment: Pimps' techniques and other circumstances that lead to street prostitution". Journal of Aggression, Maltreatment \& Trauma 15: 1-19. https://doi.org/10.1300/J146v15n02_01

Marcus, A., A. Horning, R. Curtis, J. Sanson y E. Thompson. 2014. "Conflict and Agency among sex workers and pimps: A closer look at domestic minor sex trafficking". The Annals of the American Academy of Political and Social Science 653: 225-246. https://doi. org/10.1177/0002716214521993 
Mitchell, K. J., D. Finkelhor y J. Wolak. 2010. "Conceptualizing Juvenile Prostitution as Child Maltreatment: Findings from the National Juvenile Prostitution Study". Child Maltreatment 15: 18-36. https://doi. org/10.1177/1077559509349443

Montgomery, H. 2014. "Child prostitution as filial duty? The morality of child-rearing in a slum community in Thailand". Journal of Moral Education 43: 169-182. https:// doi.org/10.1080/03057240.2014.893420

Montgomery, H. 2015. "Understanding Child Prostitution in Thailand in the 1990s". Child Development Perspectives 9:154-157. https://doi.org/10.1111/cdep.12122

Montiel Torres, O. 2011. “El oficio de padrote”. Pp. 103-133 en Trata de personas, compilado por R. Orozco. México: Instituto Nacional de Ciencias Penales.

Núñez Becerra, F. 2002. La prostitución y su represión en la ciudad de México (siglo XIX). Barcelona: Gedisa.

Orchard, T. R. 2007. "Girl, woman, lover, mother: Towards a new understanding of child prostitution among young Devadasis in rural Karnataka, India". Social Science \& Medicine 64: 2379-2390. https://doi.org/10.1016/j. socscimed.2007.02.031

Orozco, R. y E. Hernández. 2011. Del cielo al infierno en un día. México: Diamante.

Pheterson, G. 2004. "Niños/as y prostitución: reflexiones críticas sobre la legislación y la edad". Pp. 135-149 en Trabajadoras del sexo. Derechos, migraciones y tráfico en el siglo XXI, editado por R. Osborne. Barcelona: Edicions Bellaterra.

Reid, J. A. 2011. "An Exploratory Model of Girl's Vulnerability to Commercial Sexual Exploitation in Prostitution". Child Maltreatment 16: 146-157. https://doi. org/10.1177/1077559511404700

Rende Taylor, L. 2005. "Dangerous Trade-offs. The behavioral ecology of child labor and prostitution in rural northern Thailand". Current Anthropology 46: 411-431. https:// doi.org/10.1086/430079
Reyes Parra, E. 2007. Gritos en el silencio: Niñas y mujeres frente a redes de prostitución. Un revés para los derechos humanos. México: Miguel Ángel Porrúa.

Roe-Sepowitz, D. E. e Y. Katsulis. 2011. "US-Mexico Borderland Female Sex Workers: Family Responsibilities and Risks for Depression". Pp. 141-162 en Global Perspectives on Prostitution and Sex Trafficking, editado por R.L. Dalla, L.M. Baker, J. Defrain y C. Williamson. Maryland: Lexington Books.

Sano, A. 2012. "Agency and Resilience in the Sex Trade: Adolescent Girls in Rural Indramayu". Asia Pacific Journal of Anthropology 13: 21-35. https://doi.org/10.1080/144 42213.2011.636064

Servin, A. E., K. C. Brouwer, L. Gordon, T. Rocha-Jiménez y H. Staines. 2015. "Vulnerability Factors and Pathways Leading to Underage Entry into Sex Work in two Mexican-US Border Cities". Journal of Applied Research on Children: Informing Policy for Children at Risk 6: 1-10. Disponible en: http://digitalcommons.library.tmc.edu/ childrenatrisk/vol6/iss $1 / 3$

Torres Patiño, C. V. 2014. El régimen ‘abolicionista' de la prostitución en el contexto mexicano: indefinición e ideología en el no reconocimiento del trabajo sexual. Tesis de Licenciatura en Derecho presentada en el Centro de Investigación y Docencia Económicas (CIDE), Ciudad de México.

United States Department of State. 2014. Trafficking in Persons Report. https://www.state.gov/j/tip/rls/tiprpt/2014/ [Consulta 1 de octubre 2015].

Willis, B. M. y B. S. Levy. 2002. "Child prostitution: global health burden, research needs, and interventions". The Lancet 359: 1417-1422. https://doi.org/10.1016/ S0140-6736(02)08355-1

Zhang, S. X. 2011. "Woman pullers: pimping and sex trafficking in a Mexican Border City". Crime, Law and Social Change 56: 509-528. https://doi.org/10.1007/s10611011-9333-2

SIMÓN PEDRO IZCARA PALACIOS es Premio Extraordinario de Doctorado (1997/98), por la Universidad Complutense de Madrid, es profesor de Sociología en la Unidad Académica Multidisciplinaria de Ciencias, Educación y Humanidades (Universidad Autónoma de Tamaulipas, México) y miembro del Sistema Nacional de Investigadores (Nivel 3). Algunas de sus últimas publicaciones son: "Trafficking in US Agriculture", Antipode 49 (5), 1306-1328 (2017); "Migrant Smuggling on Mexico's Gulf Route: The Actors Involved", Latin American Perspectives, 16-30 (2017); "Tráfico de migrantes y terrorismo: un vínculo infundado", Política y Gobierno, 24 (2), 333-369 (2017). 